\title{
1 Proteomic Characterization of Circulating 2 Extracellular Vesicles Identifies Novel Serum Myeloma 3 Associated Markers
}

4 Sean W. Harshman ${ }^{1,2 \neq}$, Alessandro Canella ${ }^{2 \neq}$, Paul D. Ciarlariello $^{2}$, Kitty Agarwal $^{3,4}$, 5 Owen E. Branson ${ }^{3}$, Alberto Rocci ${ }^{5}$, Hector Cordero ${ }^{6}$, Mitch A. Phelps ${ }^{7}$, Erinn M. Hade ${ }^{8}$, 6 Jason A. Dubovsky ${ }^{6}$, Antonio Palumbo ${ }^{9}$, Ashley Rosko ${ }^{6}$, John C. Byrd ${ }^{6}$, Craig C. 7 Hofmeister $^{6}$, Don M. Benson, Jr. ${ }^{6}$, Michael E. Paulaitis ${ }^{4,10}$, Michael A. Freitas ${ }^{1,2 *}$, Flavia 8 Pichiorri6* $^{6 *}$

91 Department of Molecular Virology, Immunology and Medical Genetics, The Ohio State 10 University, Columbus, $\mathrm{OH}$, USA

112 Comprehensive Cancer Center, The Ohio State University, Columbus, $\mathrm{OH}, \mathrm{USA}$

123 The Ohio State Biochemistry Program, The Ohio State University, Columbus, $\mathrm{OH}$, 13 USA

144 Nanoscale Science and Engineering Center, The Ohio State University, Columbus, $15 \mathrm{OH}, \mathrm{USA}$

165 Department of Haematology, Manchester Royal Infirmary hospital, Oxford Road, 17 Manchester, UK

186 Department of Internal Medicine, Division of Hematology, The Ohio State University, 19 Columbus, OH, USA

207 Division of Pharmaceutics, College of Pharmacy, The Ohio State University, 21 Columbus, OH, USA

228 Center for Biostatistics, Department of Biomedical Informatics, The Ohio State 23 University, Columbus, $\mathrm{OH}, \mathrm{USA}$

249 Myeloma Unit, Division of Hematology, University of Turin, Azienda Ospedaliera Citta` 25 della Salute e della Scienza di Torino, 10126 Torino, Italy

2610 Department of Chemical and Biomolecular Engineering, The Ohio State University,

27 Columbus, OH, USA

$28 ¥$ These authors contributed equally to this work.

30 Extracellular Vesicles Harbor Myeloma Associated Markers

${ }^{*}$ Address reprint requests to Dr. Flavia Pichiorri The Ohio State University Medical Center, 460 West 12th Avenue, Columbus, OH 43210, USA. Phone: (614) 688-8071, Fax: (614) 688-8675, Email: flavia.pichiorri@osumc.edu or Dr. Michael A. Freitas, The Ohio State University Medical Center, 460 West 12th Avenue, Columbus, OH 43210, USA. Phone: (614) 688-8432, Fax: (614) 688-8675, Email: freitas.5@osu.edu. 


\section{ABSTRACT}

32 Multiple myeloma ( $\mathrm{MM})$ is a hematological malignancy of clonal plasma cells in the

33 bone marrow (BM). The microenvironment plays a key role in MM cell survival and drug

34 resistance through release of soluble factors, expression of adhesion molecules and

35 release of extracellular vesicles (EV). The aim of this manuscript is to use proteomic

36 profiling of EVs as a tool to identify circulating tumor associated markers in MM patients.

37 First, we characterized the EV protein content obtained from different MM cell lines.

38 Then, we established differences in protein abundance among EVs isolated from MM

39 patient serum and BM and the serum of healthy donors. These data show the Major

40 Histocompatibility Complex Class I is highly enriched in EVs of MM cell lines and MM

41 patient's serum. Next, we show that CD44 is highly expressed in the EVs isolated from

42 the corticosteroid resistant MM cell line, MM.1R. Furthermore, CD44 was found to be

43 differentially expressed in EVs isolated from newly diagnosed MM patients. Finally

44 through ELISA analysis, we establish the potential of serum CD44 as a predictive

45 biomarker of overall survival. These results support the analysis of EVs as an easily

46 accessible source for MM biomarkers. 


\section{SIGNIFICANCE}

48 Extracellular vesicles are becoming a research focus due to their roles in cancer cell

49 biology such as immune evasion, therapeutic resistance, proliferation and metastases.

50 While numerous studies of vesicle characterization and biology have been conducted in

51 many cancer models, the role of EV in MM remains relatively unstudied. Here we found

52 that EVs isolated from MM cells are enriched in MHC-1 antigen presenting complex and

53 its binding protein $\beta 2-M G$, this observation is compatible with the enhanced proteasome

54 activity of $\mathrm{MM}$ cells compared to other cancers and the ability of functional MHC-1 to

55 bind and present peptides, generated from protein degradation by the proteasome.

56 Additionally, our experiments show that CD44 is particularly enriched in the EV fraction

57 of corticosteroid resistant MM.1R cells and is differentially expressed in the EV fraction

58 of MM patients. This is of high significance due to the established role of CD44 in

59 adhesion of MM cells to BMSC and induction of IL-6, the primary cytokine for MM cell

60 survival, secretion by the BMSC. Furthermore, ELISA assays for CD44 content from the

61 serum of 254 newly diagnosed MM patients enrolled in a Phase 3 randomized trial show

62 highly variable CD44 levels and those patients with $>280 \mathrm{ng} / \mathrm{mL}$ serum CD44 showing a

63 reduced overall survival time. These results suggest the potential use of CD44 as a 64 prognostic biomarker in MM. 


\section{INTRODUCTION}

66 Multiple myeloma $(\mathrm{MM})$ is a hematological malignancy characterized by clonal plasma

67 cells (PCs) in the bone marrow (BM) and accounts for approximately 20,000 diagnoses

68 and 10,000 deaths annually in the US [1,2]. MM cells are dependent on the BM

69 microenvironment (e.g. BM stromal cells, macrophages etc) and create a network with

70 surrounding cells [3-6]. These cells play a pivotal role in the regulation of MM cell

71 survival and drug resistance by direct interactions through adhesion molecules causing

72 cell adhesion mediated drug resistance (CAM-DR) or soluble factors including

73 supportive cytokines (e.g. IL-6, IL-8, and VEGF) or exosomes (or extracellular vesicles;

74 EVs) $[7,8]$. EVs are membrane-covered cell fragments of variable size $(30-1,000 \mathrm{~nm})$,

75 which contain specific protein and RNA cargo [9-12]. BM stromal cells and MM cells can

76 mutually exchange information through soluble factors including cytokines, surface

77 molecules and EVs. Additionally, EV also have been recently reported to induce survival

78 and drug resistance in human MM cells in vitro, but the potential use of their RNA and

79 protein cargo as source of biomarkers for disease initiation and drug resistance have

80 been only partially explored $[4,8]$.

82 Recently, we established the use a global label-free quantification method to determine

83 the relative amount of proteins identified from EVs obtained from MM cell lines [13]. By

84 protein array analysis, Roccaro et al. showed that MM bone marrow myeloid stromal

85 cell (MM BM-MSC) derived EV contain increased levels of oncogenes and protein

86 kinases compared to normal BM-MSC vesicles, suggesting that EV associated protein

87 abundance can play a key role in the patho-biology of MM [4]. Although the protein 
88 array used from the authors have highlighted the presence of specific set of proteins in

89 the EV of MM BM-MSC, our global systematic proteomic analysis of EV isolated from

$90 \mathrm{MM}$ cells and from the peripheral blood of MM patients, allow the identification of

91 differential EV protein content without prior knowledge.

92

93 Here we are reporting a systematic proteomic analysis of EVs derived from MM cell

94 lines, blood from MM patients, and BM from MM patients. To characterize a specific

95 repertoire of EV-proteins from MM cells, we first measured the global EV protein content

96 of several MM cell lines and the content of circulating EV in MM primary patient samples

97 and compared the results to healthy donors. We identify the Major Histocompatibility

98 Complex Class I (MCHI) and its binding protein $\beta_{2}$-Microglobulin $\left(\beta_{2}-\mathrm{MG}\right)$ enriched in the

$99 \mathrm{EV}$ isolated from all $\mathrm{MM}$ cell lines and from $\mathrm{MM}$ patients. Because $\mathrm{MHCl}$ and $\beta_{2}-\mathrm{MG}$

100 molecules as antigen presenting complex bind antigens (peptides) generated mainly

101 from degradation of cytosolic proteins by the proteosome, it is not surprising that MM

102 cells, which for nature exhibit an increase proteosome activity, are releasing EV

103 enriched in those two molecules [14].

104

105 Previously published proteomic data has shown, that in other forms of cancer, EVs

106 isolated from drug resistant cell lines display a different protein content compared to

107 their corresponding parental cells [15-17]. We analyzed the differences in the EV protein

108 content between corticosteroid resistant (MM.1R) and sensitive (MM.1S) cell lines. We

109 identified single-chain transmembrane glycoprotein CD44 as the most differentially 
110 expressed protein in the EV of MM.1R [18-20]. Our data are consistent with the

111 previously published role of CD44 to mediate resistance to dexamethasone and

112 Lenalidomide in MM cells and with the fact that CD44 has been identified on EV derived

113 from a number of cancer cell types [21-27]. Additionally, we identified CD44 as a key

114 molecule in EVs of MM patients and confirmed its role as a prognostic marker.

\section{MATERIALS \& METHODS}

\section{ANTIBOdIES:}

118 Antibodies and dilutions used were as follows: $\beta_{2}$-Microglobulin $(1: 10,000)$, Lamin B1

119 (1:1000) (Abcam, Cambridge, MA), CD44 (1:1000, Santa Cruz Biotechnology, Santa

120 Cruz, CA) and Glyceraldehyde 3-phosphate dehydrogenase (GAPDH, Cell Signaling

121 Technology, Boston, MA). Anti-mouse \& anti-rabbit IgG-HRP (GE Healthcare,

122 Piscataway, NJ). CD44-FITC (1:20, BD Biosciences, Brea, CA). Unless otherwise noted

123 secondary antibody dilutions were 1:5000.

125 Cell Line Tissue Culture:

126 MM.1R and RPMI-8226 cell lines were obtained from American Type Culture Collection 127 (ATCC, Manassas, VA, USA). Cells were cultured as recommended by ATCC. Briefly, 128 Cell lines were maintained at $4 \times 10^{5}$ cells $/ \mathrm{mL}$ by incubation at $37^{\circ} \mathrm{C}$ with $5 \% \mathrm{CO}_{2}$ in 129 complete growth medium (RPMI-1640 with fetal bovine serum (10\%, FBS), glutamine 130 (2 $\mathrm{mM}$, GlutaMAX), penicillin-G $(50 \mathrm{U} / \mathrm{mL})$ and streptomycin $(50 \mu \mathrm{g} / \mathrm{mL}$, Life 
131 Technologies, Grand Island, NY)). For vesicle isolation, $1-2 \times 10^{8}$ cells were serum

132 starved for $48 \mathrm{~h}$ prior to vesicle enrichment.

134 Cell Line Derived Extracellular Vesicle Isolation:

$135 \mathrm{MM}$ derived extracellular vesicles were isolated as originally described by Théry et al.

136 [28]. Serum starved cells and media were differentially centrifuged at $300 \mathrm{xg}$ for $10 \mathrm{~min}$

137 at $4 \stackrel{\circ}{\circ}$ and $2000 \times \mathrm{g}$ for 20 min at $4 \stackrel{\circ}{\circ}$. Supernatant was centrifuged at $10,000 \times \mathrm{g}$ for

13830 min at $4{ }^{\circ} \mathrm{C}$ and ultracentrifuged at $100,000 \times \mathrm{g}$ for 70 min at $4{ }^{\circ} \mathrm{C}$ with vacuum. Ex

139 vivo samples were uniformly processed, fresh. Cells were spun at $300 \times \mathrm{g}$ for $10 \mathrm{~min}$ at

$1404 \stackrel{\circ}{\circ} \mathrm{C}$ and $4,500 \times \mathrm{g}$ for 20 min at $4 \stackrel{\circ}{\circ}$. Supernatant was centrifuged at $10,000 \times \mathrm{g}$ for 30

$141 \mathrm{~min}$ at $4{ }^{\circ} \mathrm{C}$ and ultracentrifuged at $100,000 \times \mathrm{g}$ for $70 \mathrm{~min}$ at $4{ }^{\circ} \mathrm{C}$ with vacuum. The

142 resulting pellets were resuspended in $1 \mathrm{~mL}$ of PBS and pooled. Pooled preparations

143 were again ultracentrifuged at $100,000 \times \mathrm{g}$ as described previously. Vesicle containing

144 pellets were frozen on dry ice and stored at $-80{ }^{\circ} \mathrm{C}$.

146 FLow CYTOMETRY:

147 Serum starved MM.1R and RPMI-8226 cell lines were analyzed for apoptosis and cell 148 death by Annexin $\mathrm{V}$ and propidium iodide flow cytometry. Approximately $1 \times 10^{5}$ serum 149 starved cells were washed with PBS and placed into Annexin V-FITC and Propidium 150 lodide staining solution (BD Biosciences, San Jose, CA) for 15 min in the dark at room 151 temperature. Cells were washed and immediately analyzed on a BD LSRII (BD 
152 Biosciences, San Jose, CA). RPMI-8226 cells were gated to eliminate confounding 153 cellular debris (Supplemental Data 1).

155 CD44-FITC staining was conducted on serum-starved cells using a Beckman Coulter 156 FC500 (Brea, CA) flow cytometer. $8 \times 10^{5}$ starved cells were stained at 1:20 in PBS for

15730 minutes on ice in the dark. Cells were washed and immediately analyzed. All 158 computational analyses were conducted using the FlowJo Software (ver. 10.0.7r2, Tree 159 Star Inc., Ashland, OR).

161 Cryo-Transmission Electron Microscopy (Cryo-TEM):

162 MM1.R and RPMI-8226 derived extracellular vesicles were prepared for cryo-TEM as 163 described previously [13]. Briefly, $4 \mu \mathrm{L}$ of vesicle suspensions were applied to glow 164 discharged lacey carbon coated copper grids (400 mesh, Pacific Grid-Tech, San 165 Francisco, CA) in a controlled environment $\left(22^{\circ} \mathrm{C}\right.$ and $95 \%$ relative humidity) using an 166 automated vitrification device (FEI Vitrobot Mark IV, FEI, Hillsboro, OR) and flash-frozen 167 in liquid ethane. Vesicles were visualized in a FEI Tecnai G2 F20 ST transmission 168 electron microscope (TEM, FEI, Hillsboro, OR) operated at $200 \mathrm{kV}$ under low dose 169 radiation. Images were captured on a Gatan Ultrascan CCD camera $(38,000 x$ 170 magnification). 
172 NANOPARTICLE Tracking ANalysis:

173 Cell lines were seeded at $3.0 \times 10^{8}$ in serum free media and grown overnight as

174 described above. Supernatant was collected and sequentially centrifuged at $3,220 \times \mathrm{g}$

175 for 10 minutes then ultracentrifuged at $10,000 \times \mathrm{g}$ for 30 minutes. Peripheral blood from

176 healthy donors and patients previously diagnosed with monoclonal gammopathy of

177 undetermined significance, smoldering and active MM were collected in EDTA

178 Vacutainer tubes (Becton Dickinson, Franklin Lakes, NJ) and sequentially centrifuged

179 with supernatant transfers after $1,000 \times \mathrm{g}$ for 10 minutes, $4500 \times \mathrm{g}$ for 15 minutes, and $18010,000 \times g$ for 30 minutes [29,30]. Remaining supernatant was homogenized then

181 diluted in PBS, as necessary. Size distribution analysis was carried out on a Nanosight

182 NS300 (Malvern Instruments Ltd., Malvern, UK) where two separate vesicle 183 preparations for each cell line and single MM patient and healthy donor preparations

184 were analyzed five times each. Batch capture was conducted on an sCMOS camera 185 with variable shutter length and frame rate, 1000 shutter setting and 400 camera gain. 186 Computational analysis was carried out on the Nanoparticle Tracking Analysis Software 187 (Malvern Instruments Ltd., Malvern, UK) version 2.3 build 0033.

189 Cell Line Vesicle Sample Preparation \& Liquid Chromatography Mass 190 SPECTROMETRY (LC-MS/MS):

191 Extracellular vesicles and cellular lysates were prepared for LC-MS/MS analysis as 192 described previously [13,31]. In triplicate, $1 \times 10^{5}$ serum starved cells or vesicle 193 isolations were dissolved in ammonium bicarbonate $(50 \mathrm{mM}$, Sigma Aldrich, St. Louis, 194 MO) with Rapigest SF surfactant (0.5\%, Waters, Milford, MA) and sequencing grade 
195 modified trypsin (800 ng, Promega, Madison, WI). Suspensions were incubated 196 overnight $(>16 \mathrm{~h}$ ) at $37 \stackrel{\circ}{\circ} \mathrm{C} .98 \%$ formic acid (Acros Organics, Geel, Belguim) was 197 added to approximately $30 \%(\mathrm{v} / \mathrm{v})$ and samples were incubated at $37{ }^{\circ} \mathrm{C}$ for $30 \mathrm{~min}$. 198 Rapigest and insoluble material was removed by centrifugation at $21,000 \times \mathrm{g}(3 \mathrm{x})$. 199 Peptides were dried and resuspended in $20 \mu \mathrm{L}$ of loading buffer (2\% acetonitrile with $2000.1 \%$ formic acid $(\mathrm{aq}))$. Peptide concentrations were estimated by the absorbance at $201280 \mathrm{~nm}$ (NanoDrop ND-1000, NanoDrop, Wilmington, DE).

203 Extracellular vesicle and cellular lysate peptides $(1-2 \mu \mathrm{g})$ were loaded onto a Dionex 204 Ultimate 3000 capillary/nano HPLC (Dionex, Sunnyvale, CA) for RP-HPLC separation. 205 Samples were directly introduced into a ThermoFisher LTQ Orbitrap XL mass 206 spectrometer (ThermoFisher, Waltham, MA) for top-5 data dependent mass analysis. 207 The Orbitrap XL was equipped with a captive spray micro/nanospray ionization source 208 for ion generation (Michrom Bioresources Inc, Auburn, CA). Peptide separations were 209 carried out on a $0.2 \mathrm{~mm} \times 150 \mathrm{~mm}$ C18 column $(5 \mu \mathrm{m}, 300 \AA$, Michrom Bioresources 210 Inc., Auburn, CA) at a flow rate of $2 \mu \mathrm{L} / \mathrm{min}$. Mobile phases: A) HPLC water (J.T. Baker,

211 Center Valley, PA):0.1 \% (v/v) formic acid and B) acetonitrile (EMD Millipore, Billerica, 212 MA):0.1\% (v/v) formic acid. The RP-HPLC separation was conducted at $2 \%$ mobile 213 phase $B$ with a linear increase to $5 \% \mathrm{~B}$ at $12 \mathrm{~min}, 15 \%$ at $40 \mathrm{~min}, 30 \%$ at $170 \mathrm{~min}, 55 \%$ 214 at $240 \mathrm{~min}, 85 \%$ at $265 \mathrm{~min}$ and $90 \%$ at $270 \mathrm{~min}$. A column wash was conducted at $90 \%$ 215 B for five minutes with a system equilibration to $2 \%$ B over 24 min. The LTQ Orbitrap $216 \mathrm{XL}$ was operated in positive ion mode with the following dynamic exclusion settings: 217 repeat count $=3$, repeat duration $=30.00$, exclusion list size $=500$, exclusion duration $=350$ 
$218 \mathrm{~s}$, exclusion mass width of $\pm 1.50 \mathrm{~m} / \mathrm{z}$. MS/MS data was searched against the UniprotKB 219 complete $\mathrm{H}$. sapiens proteome (20,237 entries as of $18 \mathrm{Sep} 12)$ using the MassMatrix 220 search engine ( $v$ 2.4.2) [32-35]. Search parameters were as follows: three missed

221 cleavages, $\mathrm{MS}^{1}$ ion tolerance of $\pm 10 \mathrm{ppm}$ and a $\mathrm{MS}^{n}$ ion tolerance of $\pm 0.8 \mathrm{Da}$. Keratin 222 identifications (cytoskeletal, epidermal and cuticle) were considered contaminant 223 proteins and removed from the analysis (listed in Supplemental Data 2-5). False 224 discovery (FDR) estimations were made using target database reversed sequences. 225 Protein IDs and spectral count data was parsed and combined using an in-house 226 python application [36]. Retention of positive protein identifications were based upon a $2275 \%$ FDR and 2 unique peptide matches or 2 max decoys.

229 Acquisition and Preparation of Primary Serum \& Bone Marrow Aspirate Derived 230 VesicLes:

231 Newly diagnosed transplant-ineligible MM patients enrolled on a Gruppo Italiano 232 Malattie EMatologiche dell'Adulto (GIMEMA) phase III clinical trial (NCT\#01063179) 233 with adequate material available for analysis, were used to study serum levels of CD44 234 [37]. On a total of 511 patient enrolled in the trial, 233 had a serum sample at diagnosis 235 that was suitable for our analysis. Clinical data at diagnosis, including ISS (International 236 Staging System) stage and FISH profile, and overall survival were available. The 237 international staging system is defined as: MGUS (serum monocolonal protein $<3 \mathrm{~g} / \mathrm{dl}$, 238 clonal bone marrow plasma cells $<10 \%$ and absence of end organ damage), SMM 239 (serum monocolonal protein $\geq 3 \mathrm{~g} / \mathrm{dl}$, clonal bone marrow plasma cells $\geq 10 \%$ and 240 absence of end organ damage) and active multiple myeloma (presences of serum or 
242 organ damage) [29]. Fluorescent in Situ Hybridization (FISH) analyses were performed

243 using purified CD138+ cells obtained from bone marrow at diagnosis [38]. Patients

244 carrying del17p13, $\mathrm{t}(4 ; 14)$, or $\mathrm{t}(14 ; 16)$ were classified as high cytogenetic risk and those

245 with no chromosomal abnormality, $\mathrm{t}(11 ; 14)$ or del(13q14) were considered standard risk

246 [39]. 13 age-matched non-cancer subjects were used as a control having had no history

247 of past or present neoplastic disease and no monoclonal gammopathy of undetermined

248 significance (MGUS). All MM patients and healthy subjects included in the analyses

249 were enrolled on IRB approved protocols and provided informed consent. For MM

250 patients and healthy subjects, serum samples were stored at $-80^{\circ} \mathrm{C}$.

252 The non-cancer, MGUS, smoldering MM (sMM) and active MM samples of plasma used

253 to extract extracellular vesicles were derived from The Buckeye Surveillance, Contact 254 and Research MM Registry (IRB \#2010C0126). The plasma extracted from cancer free 255 patients' significant others consented for peripheral blood sample banking, allowing for 256 an age-matched, were also used. EVs were isolated from serum by differential 257 centrifugation/filtration from a total of $10 \mathrm{~mL}$ of blood (serum collection tube) obtained 258 from 40 individual MM patients and 15 individual healthy donors, as previously 259 published [13]. 


\section{Serum and Bone Marrow Vesicle Sample Preparation \& LC-MS/MS:}

262 Healthy donor serum and MM serum/bone marrow derived vesicles were prepared for

263 LC-MS/MS analysis as described above for cell line derived vesicles. 1-2 $\mu \mathrm{g}$ of vesicle

264 peptides were separated on a Thermo Scientific Easy-nLC II (Thermo Scientific, 265 Waltham, MA) nano-HPLC system coupled to a hybrid Orbitrap Elite ETD (Thermo 266 Scientific, Waltham, MA) mass spectrometer for mass analysis as described by Yi et al. 267 [40]. In-line desalting was accomplished using a reversed-phase trap column $(100 \mu \mathrm{m} \times$ $26820 \mathrm{~mm}$, Magic C18 AQ, $5 \mu \mathrm{m}, 200 \AA$ A, Michrom Bioresources, Auburn, CA) followed by 269 peptide separation on a reversed-phase column $(75 \mu \mathrm{m} \times 250 \mathrm{~mm}$, Magic C18 AQ, 5

$270 \mu \mathrm{m} 100 \AA$ A Michrom Bioresources, Auburn, CA) directly mounted on the electrospray ion

271 source. A 90-minute gradient from $7 \%$ to $35 \%$ acetonitrile in $0.1 \%$ formic acid was

272 conducted at a flow rate of $300 \mathrm{~nL} /$ minute. The heated capillary temperature was set to

$273300{ }^{\circ} \mathrm{C}$ and a spray voltage of $2,250 \mathrm{~V}$ was applied to the electrospray tip. The Orbitrap

274 Elite instrument was operated in top 20 data-dependent mode, switching automatically

275 between MS survey scans in the Orbitrap (AGC target value 1,000,000, resolution 276240,000 , and injection time 250 milliseconds) with MS/MS spectra acquisition in the 277 linear ion trap (AGC target value of 10,000 and injection time 100 milliseconds). 278 Collision-induced dissociation (CID) used normalized collision energy of $35 \%$. Selected 279 ions were dynamically excluded for 30 seconds with a list size of 500 and exclusion 280 mass width of $\pm 10 \mathrm{ppm}$. MS/MS data was searched as described above for cell line 281 vesicles. Keratin identifications are provided in Supplemental Data 8-11. 


\section{Label-Free Relative Quantitation:}

284 Label-free relative quantitation was performed using the approach originally described

285 by Liu et al. and Colinge et al. [41,42]. Spectral counts do not include modified, or semi-

286 tryptic peptides and shared peptides from multiple protein isoforms contribute equally to

287 each isoform. The MS/MS data for each sample along with biological and/or technical

288 replicates was combined using an in-house developed application into parsimonious

289 protein lists. Spectral count quantitation was performed on the top protein matches with

290 distinct peptide sequences (two or more) in at least a single sample and a protein score

291 above the decoy threshold. The decoy threshold was set by the protein score for the

292 third decoy ID or the decoy score that exceeds the false discovery rate of $5 \%$.

293 Quantitation of spectral counts was limited to those proteins with at least one 294 observation in each replicate with a minimum of 5 total spectral counts. The spectral 295 count data and FDRs estimations are provided in Supplemental Data 2-5 \& 8-11. Raw 296 MS/MS spectral counts were adjusted to center, normalize and cluster across the arrays 297 and genes using the average linkage function of the Cluster 3.0 software (C Clustering 298 Library 1.50). Visualization of clustered spectral counts was conducted using the Java 299 Tree View (ver. 1.1.6r2) software.

301 Computational AnNotations And Bioinformatics:

302 2-way and 4-way Venn diagrams of protein identifications were generated using the 303 BioVenn (http://www.cmbi.ru.nl/cdd/biovenn/) and Venny web applications 304 (http://bioinfogp.cnb.csic.es/tools/venny/) [43]. Gene ontological annotations were 
305 determined by the use of the PANTHER Classification System 306 (http://www.pantherdb.org/) [44,45].

\section{IMMUNOBLOTTING:}

309 Extracellular vesicle preparations and cell pellets were lysed using $50 \mathrm{mM}$ Tris at $\mathrm{pH} 7.5$, $310150 \mathrm{mM} \mathrm{NaCl}, 10 \%$ Glycerol, $1.0 \%$ NP-40, $0.1 \%$ SDS supplemented with protease 311 and phosphatase inhibitors. Protein concentrations were estimated by Bradford assay 312 and equivalent quantities of the lysates were run on $4-15 \%$ Tris-HCI SDS-PAGE TGX 313 gels (Bio-Rad, Richmond, CA). Proteins were transferred to nitrocellulose and blotted 314 for CD44, $\beta_{2}-M G$, and GAPDH using either the ECL Western Blotting Detection 315 Reagents (GE Healthcare, Piscataway, NJ) or SuperSignal West Femto Kit (Pierce 316 Biotechnology, Rockford, IL).

\section{ENZYME-LINKED IMMUNOSORBENT ASSAY (ELISA):}

319 The ELISA performed for CD44 content in primary serum was conducted as described 320 by the manufacturer (Abcam, Cambridge, MA). Briefly, primary serum was diluted 1:40 321 in Standard Diluent Buffer. In duplicate onto a 96-well plate, $100 \mu \mathrm{L}$ of each sample, 322 each standard and $1 \mathrm{x}$ control solution was added to the appropriate well and incubated 323 for $1 \mathrm{~h}$. All incubations were conducted at room temperature unless otherwise noted. 324 The plate was washed, biotinylated anti-CD44 was added to each well and solutions 325 were incubated for $30 \mathrm{~min}$ (repeated). Added $100 \mu \mathrm{L}$ 1x Streptavidin-HRP and allowed 326 to stand for $30 \mathrm{~min}$. In the dark, $100 \mu \mathrm{L}$ of Chromogen TMB substrate was added to 
327 each well and incubated for $15 \mathrm{~min} .100 \mu \mathrm{L}$ of Stop Reagent was added and

328 absorbance at $450 \mathrm{~nm}$ was read on a spectrophotometer. Calculations for CD44 content

329 were made based upon the standard curve and sample dilution factors.

331 Statistical Analysis:

332 Overall survival was defined as the time from study enrollment to the time of death.

333 Patients who did not die during the study follow up period were censored at the end of

334 study follow up or the date of last follow up. Associations between baseline disease

335 characteristics and overall survival were estimated through Cox proportional hazards

336 models (Supplemental Data 19) and Kaplan and Meier. The assumption of proportional

337 hazards was assessed through tests of the Schoenfeld residuals with no violations

338 observed. All reported p-values are two-sided and analyses were performed using the 339 statistical program Stata (version 13, StataCorp, College Station, TX).

341 RESULTS

342 MHC Class I and Beta 2 -Microglobulin are Commonly Enriched in MM Cell Lines 343 Derived EV

344 To further corroborate our previously published data in which we have shown MM cell

345 lines produce EV with similar physical characteristics, we extended our proteomic 346 analysis to the EV population derived from an additional MM cell line, RPMI-8226.

347 Additionally, we analyzed vesicles from the dexamethasone (corticosteroid) resistant, 348 MM.1R, cells. These cells are derived from the parental, MM.1S, cell line, that we 
349 previously analyzed and published [13]. Annexin V/Propidium lodide (Annexin/PI)

350 staining was performed in the MM cells used as source of EV. Less than $2 \%$ apoptosis

351 was observed, supporting the idea that EV are not the result of programmed cell death

352 (Supplemental Figure 1). Cryo-Transmission Electron Microscopy (Cryo-TEM) and

353 Nanoparticle Tracking Analsysis (NTA) show that the EV diameters are approximately

35475 and $175 \mathrm{~nm}$ in all MM cell lines we analyzed (Figures 1A \& 1B) [13].

356 To further assess whether the EV isolated from these two cell lines could also be

357 enriched in specific secreted proteins, a shotgun proteomics approach was applied to

358 the cellular and derived EV fraction for protein identification [13]. Comparison of the

359 identified MM EV proteins from the current study (RPMI-8226 and MM.1R) and our

360 previous study (MM.1S and U266) shows a large number of overlapping proteins (339)

361 between the four data sets (Figure 1C \& Supplemental Data 2-5). Of the 497 total

362 proteins identified, 77 were uniquely found, when compared to global lysate protein IDs,

363 in the EVs of the 4 MM cell lines. Gene ontological analysis of the 77 proteins show a

364 diverse range of molecular functions, biological processes, cellular components and

365 protein classes (Supplemental 6). Interestingly, these data show that the $\mathrm{MHCl}$ and its

366 associated binding protein, $\beta_{2}-\mathrm{MG}$, were among the most abundant communally

367 enriched proteins across the $4 \mathrm{MM}$ cell line derived $\mathrm{EV}$ analyzed (Figure 1D, 1E \&

368 Supplemental Data 7). In addition, $\beta_{2}-M G$ expression in the EV fraction was correlated

369 with EV-MHCl protein levels (Figures 1D \& 1E). These results support the hypothesis

370 that an active antigen-presenting complex is secreted through EV, as previously

371 reported in other cellular systems [46]. The observed enrichments could be associated 
373 of myeloma cells compared to other forms of cancer [14].

375 MHCl and B $B_{2}$-MG Are EnRiched in EV Isolated from the Serum of Newly Diagnosed 376 MM PATIENTS

377 The clinical translation of in vitro models is of utmost importance. Hence, we assessed 378 whether as in the case of cell lines, we could enrich a uniform EV population from the 379 serum of different donors. NTA analysis was performed to evaluate the size distributions 380 of the patient derived serum EVs of symptomatic MM (active MM), asymptomatic MM 381 termed smoldering MM (sMM), premalignant MM termed Monoclonal Gammopathy of

382 Undetermined Significance (MGUS) and from healthy donors. As shown in Figure 2A, 383 the patient derived EV are between $50-100 \mathrm{~nm}$ in diameter with no statistically 384 significant difference in numbers and size distributions between any of the groups by 385 one-way ANOVA ( $\mathrm{p}=0.4088$ ). The NTA analysis provides results that are consistent with 386 previous reports for serum derived EV diameters [47]. LC-MS/MS analysis, which was 387 previously used for EV derived MM cell lines, was conducted on the EVs isolated from 388 two newly diagnosed MM patients by collecting serum and bone marrow aspirates. The 389 protein identifications and spectral count data from each sample type are provided in 390 Supplemental Data 8-11. A comparison of the protein identifications by LC-MS/MS 391 show many ubiquitously identified proteins (343) in each sample type (Figure 2B). 392 These results are similar to those observed in the cell line EV data (Figure 1C). 393 Clustering analysis confirmed enrichment of $\mathrm{MHCl}$ in the serum and in the $\mathrm{BM}$ of $\mathrm{MM}$ 394 patients compared to healthy donors (Supplemental Data 11). Additionally, $\beta_{2}-M G$ was 
395 identified in these samples, but spectral counts were below the filter limits for the 396 clustering analysis as described in the methods section. Immunoblot analysis validates

397 enrichment of $\mathrm{MHCl}$ and $\beta_{2}-\mathrm{MG}$ in the $\mathrm{EV}$ isolated from newly diagnosed $\mathrm{MM}$ patients

398 compared to healthy donors with $\beta_{2}-\mathrm{MG}$ expression correlated with EV-MHCl protein 399 levels (Figure 2C).

401 CD44 is EnRiched in the EVs Isolated from DeXamethasone Resistant MM Cells

402 To assess whether MM derived EVs could harbor proteins associated with mechanisms 403 of drug resistance, the EV and cellular protein contents from the dexamethasone 404 resistant MM cells, MM.1R, were compared with those obtained from the sensitive 405 parental cell line, MM.1S. By LC-MS/MS, we observed that the EV fraction obtained 406 from MM.1R cells were significantly enriched in the main receptor of hyaluronic acid, 407 CD44. Interestingly, CD44 expression has been extensively associated with 408 mechanisms of drug resistance, not only in $\mathrm{MM}$, but also in other forms of cancer $409[26,27,48,49]$. CD44 protein enrichment was observed in the MM.1R derived EV, but no 410 differences were found in the cellular protein levels of CD44 between sensitive (MM.1S) 411 and resistant cells (MM.1R, Figure 3A \& 3B). Immunoblot analysis of the EVs isolated 412 from the MM cell lines confirms the enrichment of CD44 in the MM.1R EVs compared to 413 other MM cell line's EVs, including the MM.1S cell derived EVs. The enrichment of EV414 CD44 from the MM.1R cells, compared to MM.1S, correlates to an increase of CD44 on 415 the cell surface of MM.1R compared to MM.1S cells (Figure 3D). Because GAPDH 416 seems to be differentially present in EV fraction, Lamin B1 was used to assess the 417 purity of our EV preparation compared to the cellular fraction. Furthermore, $\beta_{2}-\mathrm{MG}$ 
419 support the hypothesis that membrane CD44 could be responsible of mechanisms of 420 drug resistance, as also previously suggested by Vincent et al [50].

Identification of Extracellular Vesicles as the Primary location of Serum CD44

\section{IN MM PATIENTS}

424 The glycoprotein CD44 has been shown to play a key role in various cellular functions in 425 normal cells, such as signaling, cell adhesion and microenvironmental interactions [51]. 426 However in hematological malignancies, CD44 has been implicated in increased 427 invasiveness, cancer cell trafficking and resistance to apoptosis [51]. In our study, CD44 428 was identified not only in the EV of MM resistant cells but also in primary patients with 429 variable abundance (Figure 3 \& Supplemental Data 12). Through immunoblotting, we 430 show CD44 is contained in the EVs isolated, not only from MM cells, but also from MM 431 patient serum (32 newly diagnosed MM patients and 13 healthy individuals). Figure 4A 432 shows a representative blot for CD44, from whole serum, with additional blots provided 433 in Supplemental Data 13. These data illustrate the variable expression of CD44 in 434 several MM patients' serum when compared to healthy individuals. To confirm the 435 localization of CD44 to the EV of the MM patient serum, the serum of two patients 436 showing high CD44 levels, in labeled 1 \& 2 in Figure 4A, was depleted of EV by 437 ultracentrifugation. Figure 4B shows the immunoblot for CD44 from the samples not 438 depleted (+) and depleted (-) of EV. These results demonstrate a reduced CD44 content 439 when serum is depleted of EVs, suggesting the CD44 in the patent's serum is mainly 440 localized to the EV. The depletion of CD44 from the serum, by removing the EV, was 
441 further validated by an ELISA analysis (Figure 4C). Similarly to Figure 4B, the ELISA

442 results, of those samples labeled 1 \& 2 in Figure 4A not depleted and depleted of EV,

443 show a reduction in CD44 content in the serum depleted of EV. Collectively, these

444 results show the CD44 content of MM patient serum is primarily localized to the 445 circulating EV fraction.

447 Serum CD44 Levels in Newly Diagnosed MM Patients is Associated with Overall 448 SURVIVAL

449 Our results indicated increased expression levels of CD44 in the EVs isolated from 450 corticosteroid resistant MM cells, possibly suggesting a role in drug resistance. EV 451 associated CD44 is differentially expressed in the serum of MM patients and increased 452 expression of specific CD44 isoforms on the surface of MM cells by 453 immunohistochemistry has been correlated with poor prognosis and decreased overall 454 survival [52]. Hence, we hypothesize that serum CD44 could be used as a prognostic 455 marker in MM patients, which are normally treated in combinatorial therapy as standard 456 of care. ELISA assays were conducted for CD44 from the serum collected from 13 457 healthy individuals and 233 newly diagnosed MM patients prospectively enrolled in a 458 Phase 3 clinical trial including corticosteroid treatment. Patients received Velcade459 Melphalan-Prednisone (VMP) or Velcade-Melphalan-Prednisone-Thalidomide 460 followed by maintenance with Velcade-Thalidomide (VMPT-VT) through the parent 461 clinical trial (NCT\#01063179). CD44 values for the healthy individuals range from 462 235.69-122.32 $\mathrm{ng} / \mathrm{mL}$ while the MM patients have a much wider range, 6.43-637.51 $463 \mathrm{ng} / \mathrm{mL}$ (Figure 5A \& 5B, Supplemental Data 14). The statistical analysis of the ELISA 
464 data shows no statistical difference between the means of MM patients $(143.0 \mathrm{ng} / \mathrm{mL})$

465 and healthy donor $(166.8 \mathrm{ng} / \mathrm{mL})$ CD44 levels based on a student's t-test $(p=0.5070)$.

466 However, these data show that the CD44 levels in the MM patient serum are highly

467 variable and maybe useful for stratifying MM patients at diagnosis. In fact, one approach

468 to improve risk prognostication in patients with $\mathrm{MM}$ is to use new technologies to stratify

469 patients based on distinct outcomes [53]. Outcome prediction for most patients is based

470 on the International Staging System (ISS) and the presence or absence of specific

471 fluorescent in-situ hybridization abnormalities. Additional biomarkers are necessary to

472 improve precision. Our data show that CD44, which has been involved in mechanisms

473 of Myeloma drug resistance and therapeutic outcome, is present in soluble form in the

474 serum of MM patients and it can be released from MM cells through EV [27,54].

475 Therefore, we decided to evaluate its possible utility as non-invasive biomarker. To

476 address this question, we measured serum CD44 levels in a large cohort of newly

477 diagnosed MM patients who were uniformly treated and followed, correlating serum

478 CD44 levels with clinical outcome to test their prognostic impact in a multivariate model 479 [37].

480

481 Of the 233 newly diagnosed patients analyzed for CD44, 87 died during the course of 482 follow up with a five-year overall survival of $56.6 \%(95 \% \mathrm{Cl}: 44.8 \%-66.8 \%)$. In age483 adjusted models, a strong increased risk of death was associated with increased ISS 484 stage, elevated $\beta_{2}-M G$ and increased serum CD44 (Figure 5C). When modeled 485 together, following multiple imputation of missing ISS stage, serum CD44 and ISS stage 486 remain significantly associated with overall survival (Figure $\mathbf{5 C}$ ). The risk of death 
487 increased significantly when CD44 was greater than the maximum value in normal 488 controls of $280 \mathrm{ng} / \mathrm{mL}$ after adjusting for age and stage (Figure 5D, adjusted hazard 489 ratio: $3.00,95 \% \mathrm{Cl}: 1.36-6.45)$. Collectively, these data establish serum CD44 as a 490 potential biomarker of overall survival in multiple myeloma.

\section{DISCUSSION}

493 EVs are becoming a research focus due to their roles in cancer cell biology such as

494 immune evasion, therapeutic resistance, proliferation and metastases (recently 495 reviewed in $[55,56])$. EV from MM BM-MSCs contain miRNA that are taken up by MM 496 cells and influence tumor growth and dissemination, and it is not known if this is bi497 directional and if $\mathrm{MM}$ cells derived $\mathrm{EV}$ are also responsible to affect the cancer 498 microenvironment towards their survival advantage [4]. While numerous studies of 499 vesicle characterization and biology have been conducted in many cancer models, the 500 role of EV in MM remains relatively unstudied. Roccaro et al. recently established 501 implications for the importance of EV released by BMSC in MM biology through 502 characterization of the protein content of the MM BM-MSC derived vesicles by protein 503 array [4]. They found that when compared to normal BM-MSC, the MM derived BM-

504 MSC EV had higher expression of oncogenes and protein kinases [4]. They establish a 505 functional relevance of EV to the MM microenvironment, however these results focus 506 only on the BM-MSC derived vesicles and the protein spectrum analyzed was limited. 
508 Here we found that EVs isolated from MM cells are enriched in MHC-1 antigen 509 presenting complex and its binding protein $\beta_{2}-M G$, this observation is compatible with

510 the enhanced proteasome activity of MM cells compared to other cancers and the ability

511 of functional MHC-1 to bind and present peptides, generated from protein degradation

512 by the proteasome [14]. A proportional enrichment was also observed in the peripheral

513 blood of MM patients for $\mathrm{MHCl}$ when compared to healthy donors by LC-MS/MS and

$514 \mathrm{MHCl}$ and $\beta_{2}-\mathrm{MG}$ immunoblot analysis. It is well known that the serum level of $\beta_{2}-\mathrm{MG}$ is

515 an important prognostic factor in MM. However, vesicle $\beta_{2}-M G$ represents only a small

516 percentage (data not shown) of the total serum $\beta_{2}-M G$, suggesting that two separate $\beta_{2^{-}}$

517 microglobulin populations coexist in the serum of patients. However, further studies are

518 required to investigate their different biological functions.

520 CD44 has been identified on extracellular vesicles derived from a number of cell types 521 and locations, including B cells, bladder and colorectal cancer cells, saliva and urine 522 [21-25]. Specifically in MM, CD44 causes adhesion of MM cells to BMSC and induction

523 of IL-6, the primary cytokine for MM cell survival, secretion by the BMSC [51].

524 Additionally, specific alternatively spliced variants of CD44, such as CD44v9, have been 525 studied on MM cells linking CD44 expression to advancing disease status and shorter 526 overall survival [52].

528 Our experiments show that CD44 is particularly enriched in the EV fraction of 529 corticosteroid resistant MM.1R cells and is differentially expressed in the EV fraction of 
530 MM patients. Our serum depletion experiments show that CD44 is primarily localized to

531 the peripheral EV in MM patients. Furthermore, ELISA assays for CD44 content from

532 the serum of 254 newly diagnosed MM patients enrolled in a Phase 3 randomized trial

533 show highly variable CD44 levels [13]. Additionally, those patients with $>280 \mathrm{ng} / \mathrm{mL}$

534 serum CD44 showed a reduced overall survival time. These results suggest the

535 potential use of CD44 as a prognostic biomarker. Because previously published data

536 have specifically associated CD44 as survival protein for MM cells responsible in

537 increasing MM cell adhesion to BMSC and in inducing IL-6 secretion by the BMSC [51],

538 it is not surprising that increased level of soluble CD44 can be associated with decrease

539 survival of MM patients. The presence of CD44 in EV of other cancers highlights the key

540 role of this protein as hallmark of cancerous EV. Moreover, its differential expression

541 between healthy subjects and MM patients suggests it could be a selective target for

542 cancer vesicles.

\section{ACKNOWLEDGEMENTS}

545 This work was supported by the Ohio State University Pelotonia Fellowship Program

546 (A.R.), by Ohio State University Pelotonia Idea grant (F.P., D.B.), in part from NIH

547 grants (R01 CA107106, P01 CA124570 and RC2 AG036559), NSF (EEC-0425626 and

548 EEC-019790) and funded in part by Multiple Myeloma Opportunities for Research \&

549 Education (MMORE) and in part from Sidney Kimmel Foundation Scholar Award (F.P.). 
551 The cryo-TEM data were obtained at the TEM facility at the Liquid Crystal Institute, Kent

552 State University, supported by the Ohio Research Scholars Program Research Cluster 553 on Surfaces in Advanced Materials. The authors thank Dr. Min Gao for technical 554 support provided for the TEM experiments. 
American Cancer Society, Cancer Facts \& Figures, (2011) 1-68. $<$ http://www.cancer.org/acs/groups/content/@epidemiologysurveilance/docu ments/document/acspc-031941.pdf>.

W.M. Kuehl, P.L. Bergsagel, Multiple myeloma: evolving genetic events and host interactions, Nat Rev Cancer. 2 (2002) 175-187.

M.R. Reagan, I.M. Ghobrial, Multiple Myeloma-Mesenchymal Stem Cells: Characterization, Origin, and Tumor-Promoting Effects, Clin Cancer Res. 18 (2012) 342-349.

A.M. Roccaro, A. Sacco, P. Maiso, A.K. Azab, Y.-T. Tai, M. Reagan, et al., BM mesenchymal stromal cell-derived exosomes facilitate multiple myeloma progression, J Clin Invest. 123 (2013) 1542-1555.

A. Vacca, D. Ribatti, A.M. Roccaro, A. Frigeri, F. Dammacco, Bone marrow angiogenesis in patients with active multiple myeloma, Semin Oncol. 28 (2001) 543-550.

A. Mahindra, T. Hideshima, K.C. Anderson, Multiple myeloma: biology of the disease, Blood Rev. 24 (2010) S5-S11.

J.S. Damiano, A.E. Cress, L.A. Hazlehurst, A.A. Shtil, W.S. Dalton, Cell adhesion mediated drug resistance (CAM-DR): role of integrins and resistance to apoptosis in human myeloma cell lines, Blood. 93 (1999) 1658-1667.

J. Wang, A. Hendrix, S. Hernot, M. Lemaire, E. De Bruyne, E. Van Valckenborgh, et al., Bone marrow stromal cell-derived exosomes as communicators in drug resistance in multiple myeloma cells, Blood. 124 (2014) 555-566.

A.V. Vlassov, S. Magdaleno, R. Setterquist, R. Conrad, Exosomes: current knowledge of their composition, biological functions, and diagnostic and therapeutic potentials, BBA-Gen Subjects. 1820 (2012) 940-948.

K. Denzer, M.J. Kleijmeer, H.F. Heijnen, W. Stoorvogel, H.J. Geuze, Exosome: from internal vesicle of the multivesicular body to intercellular signaling device, J Cell Sci. 113 (2000) 3365-3374.

C. Théry, M. Boussac, P. Véron, P. Ricciardi-Castagnoli, G. Raposo, J. Garin, et al., Proteomic analysis of dendritic cell-derived exosomes: a secreted subcellular compartment distinct from apoptotic vesicles, J Immunol. 166 (2001) 7309-7318.

R. Wubbolts, R.S. Leckie, P.T.M. Veenhuizen, G. Schwarzmann, W. Möbius, J. Hoernschemeyer, et al., Proteomic and biochemical analyses of human B cell-derived exosomes. Potential implications for their function and multivesicular body formation, J Biol Chem. 278 (2003) 10963-10972.

S.W. Harshman, A. Canella, P.D. Ciarlariello, A. Rocci, K. Agarwal, E.M. Smith, et al., Characterization of Multiple Myeloma Vesicles by Label-Free Relative Quantitation, Proteomics. 13 (2013) 3013-3029.

C.M. Edwards, S.T. Lwin, J.A. Fowler, B.O. Oyajobi, J. Zhuang, A.L. Bates, et al., Myeloma cells exhibit an increase in proteasome activity and an enhanced response to proteasome inhibition in the bone marrow microenvironment in vivo, Am J Haematol. 84 (2009) 268-272. 
601

602

603

604

605

606

607

608

609

610

611

612

613

614

615

616

617

618

619

620

621

622

623

624

625

626

627

628

629

630

631

632

633

634

635

636

637

638

639

640

641

642

643

644

645

646

[15] D. Pokharel, M.P. Padula, J.F. Lu, J.L. Tacchi, F. Luk, S.P. Djordjevic, et al., Proteome analysis of multidrug-resistant, breast cancer-derived microparticles, J Extracell Vesicles. 3 (2014) 24384.

[16] D.-Y. Choi, S. You, J.H. Jung, J.C. Lee, J.K. Rho, K.Y. Lee, et al., Extracellular vesicles shed from gefitinib-resistant nonsmall cell lung cancer regulate the tumor microenvironment, Proteomics. 14 (2014) 1845-1856.

[17] X. Ma, Z. Chen, D. Hua, D. He, L. Wang, P. Zhang, et al., Essential role for TrpC5-containing extracellular vesicles in breast cancer with chemotherapeutic resistance, Proc Natl Acad Sci USA. 111 (2014) 63896394.

[18] T. Ahrens, V. Assmann, C. Fieber, C.C. Termeer, P. Herrlich, M. Hofmann, et al., CD44 is the principal mediator of hyaluronic-acid-induced melanoma cell proliferation, J Invest Dermatol. 116 (2001) 93-101.

[19] T. Ahrens, J.P. Sleeman, C.M. Schempp, N. Howells, M. Hofmann, H. Ponta, et al., Soluble CD44 inhibits melanoma tumor growth by blocking cell surface CD44 binding to hyaluronic acid, Oncogene. 20 (2001) 3399-3408.

[20] D. Naor, R.V. Sionov, D. Ish-Shalom, CD44: structure, function, and association with the malignant process, Adv Cancer Res. 71 (1997) 241319.

[21] S.I. Buschow, B.W.M. van Balkom, M. Aalberts, A.J.R. Heck, M. Wauben, W. Stoorvogel, MHC class II-associated proteins in B-cell exosomes and potential functional implications for exosome biogenesis, Immunol Cell Biol. 88 (2010) 851-856.

[22] J.L. Welton, S. Khanna, P.J. Giles, P. Brennan, I.A. Brewis, J. Staffurth, et al., Proteomics analysis of bladder cancer exosomes, Mol Cell Proteomics. 9 (2010) 1324-1338.

[23] D.-S. Choi, J.O. Park, S.C. Jang, Y.J. Yoon, J.W. Jung, D.-Y. Choi, et al., Proteomic analysis of microvesicles derived from human colorectal cancer ascites, Proteomics. 11 (2011) 2745-2751.

[24] S. Mathivanan, J.W.E. Lim, B.J. Tauro, H. Ji, R.L. Moritz, R.J. Simpson, Proteomics analysis of A33 immunoaffinity-purified exosomes released from the human colon tumor cell line LIM1215 reveals a tissue-specific protein signature, Mol Cell Proteomics. 9 (2010) 197-208.

[25] M. Gonzalez-Begne, B. Lu, X. Han, F.K. Hagen, A.R. Hand, J.E. Melvin, et al., Proteomic Analysis of Human Parotid Gland Exosomes by Multidimensional Protein Identification Technology (MudPIT), J Proteome Res. 8 (2009) 1304-1314.

[26] C. Ohwada, C. Nakaseko, M. Koizumi, M. Takeuchi, S. Ozawa, M. Naito, et al., CD44 and hyaluronan engagement promotes dexamethasone resistance in human myeloma cells, Eur J Haematol. 80 (2008) 245-250.

[27] C.C. Bjorklund, V. Baladandayuthapani, H.Y. Lin, R.J. Jones, I. Kuiatse, H. Wang, et al., Evidence of a role for CD44 and cell adhesion in mediating resistance to lenalidomide in multiple myeloma: therapeutic implications, Leukemia. 28 (2014) 373-383.

[28] C. Théry, S. Amigorena, G. Raposo, A. Clayton, Isolation and characterization of exosomes from cell culture supernatants and biological 
fluids, Curr Protoc Cell Biol. Chapter 3 (2006) Unit 3.22.

International Myeloma Working Group, Criteria for the classification of monoclonal gammopathies, multiple myeloma and related disorders: a report of the International Myeloma Working Group, Br J Haematol. 121 (2003) 749-757.

S.V. Rajkumar, Multiple myeloma: 2012 update on diagnosis, riskstratification, and management, Am J Haematol. 87 (2012) 78-88.

J.P. Shapiro, S. Biswas, A.S. Merchant, A. Satoskar, C. Taslim, S. Lin, et al., A quantitative proteomic workflow for characterization of frozen clinical biopsies: laser capture microdissection coupled with label-free mass spectrometry, J Proteomics. 77 (2012) 433-440.

H. Xu, M.A. Freitas, A mass accuracy sensitive probability based scoring algorithm for database searching of tandem mass spectrometry data, BMC Bioinformatics. 8 (2007) 133.

$\mathrm{H}$. Xu, L. Yang, M.A. Freitas, A robust linear regression based algorithm for automated evaluation of peptide identifications from shotgun proteomics by use of reversed-phase liquid chromatography retention time, BMC Bioinformatics. 9 (2008) 347.

$\mathrm{H}$. Xu, L. Zhang, M.A. Freitas, Identification and characterization of disulfide bonds in proteins and peptides from tandem MS data by use of the MassMatrix MS/MS search engine, J Proteome Res. 7 (2008) 138-144.

$\mathrm{H}$. Xu, M.A. Freitas, Monte carlo simulation-based algorithms for analysis of shotgun proteomic data, J Proteome Res. 7 (2008) 2605-2615.

B. Zhang, M.C. Chambers, D.L. Tabb, Proteomic parsimony through bipartite graph analysis improves accuracy and transparency, J Proteome Res. 6 (2007) 3549-3557.

A. Palumbo, Bortezomib-Melphalan-Prednisone-Thalidomide Followed by Maintenance With Bortezomib-Thalidomide Compared With BortezomibMelphalan-Prednisone for Initial Treatment of Multiple Myeloma: A Randomized Controlled Trial, J Clin Oncol. 28 (2010) 5101-5109.

F.M. Ross, The $T(14 ; 20)$ Is a Poor Prognostic Factor in Myeloma but Is Associated with Long-Term Stable Disease in Monoclonal Gammopathies of Undetermined Significance, Haematologica. 95 (2010) 1221-1225.

N.C. Munshi, Consensus recommendations for risk stratification in multiple myeloma: report of the International Myeloma Workshop Consensus Panel 2, Blood. (2011) 4694-4700.

E.C. Yi, H. Lee, R. Aebersold, D.R. Goodlett, A microcapillary trap cartridgemicrocapillary high-performance liquid chromatography electrospray ionization emitter device capable of peptide tandem mass spectrometry at the attomole level on an ion trap mass spectrometer with automated routine operation, Rapid Commun Mass Spectrom. 17 (2003) 2093-2098.

H. Liu, R.G. Sadygov, J.R. Yates, A model for random sampling and estimation of relative protein abundance in shotgun proteomics, Anal Chem. 76 (2004) 4193-4201.

J. Colinge, D. Chiappe, S. Lagache, M. Moniatte, L. Bougueleret, Differential proteomics via probabilistic peptide identification scores, Anal Chem. 77 
[43] T. Hulsen, J. de Vlieg, W. Alkema, BioVenn - a web application for the comparison and visualization of biological lists using area-proportional Venn diagrams, BMC Genomics. 9 (2008) 488.

[44] P.D. Thomas, A. Kejariwal, M.J. Campbell, H. Mi, K. Diemer, N. Guo, et al., PANTHER: a browsable database of gene products organized by biological function, using curated protein family and subfamily classification, Nucleic Acids Res. 31 (2003) 334-341.

700

701

702

[45] H. Mi, Q. Dong, A. Muruganujan, P. Gaudet, S. Lewis, P.D. Thomas, PANTHER version 7: improved phylogenetic trees, orthologs and collaboration with the Gene Ontology Consortium, Nucleic Acids Res. 38 (2010) D204-10.

704

705

706

707

708

709

710

711

712

713

714

715

716

717

718

719

720

721

722

723

724

725

726

727

728

729

730

731

732

733

734

735

736

T. Vraetz, T.H. Ittel, M.G. van Mackelenbergh, P.C. Heinrich, H.G. Sieberth, L. Graeve, Regulation of $\beta 2$-microglobulin expression in different human cell lines by proinflammatory cytokines, Nephrol Dial Transplant. 14 (1999) 2137-2143.

[47] Y. Tanaka, H. Kamohara, K. Kinoshita, J. Kurashige, T. Ishimoto, M. Iwatsuki, et al., Clinical impact of serum exosomal microRNA-21 as a clinical biomarker in human esophageal squamous cell carcinoma, Cancer. 119 (2013) 1159-1167.

[48] X. Yang, A.K. lyer, A. Singh, L. Milane, E. Choy, F.J. Hornicek, et al., Cluster of Differentiation 44 Targeted Hyaluronic Acid Based Nanoparticles for MDR1 siRNA Delivery to Overcome Drug Resistance in Ovarian Cancer, Pharm Res. 32 (2015) 2097-2109.

[49] C. Yoon, D.J. Park, B. Schmidt, N.J. Thomas, H.-J. Lee, T.S. Kim, et al., CD44 expression denotes a subpopulation of gastric cancer cells in which Hedgehog signaling promotes chemotherapy resistance, Clin Cancer Res. 20 (2014) 3974-3988.

[50] T. Vincent, N. Mechti, IL-6 regulates CD44 cell surface expression on human myeloma cells, Leukemia. 18 (2004) 967-975.

[51] M.K. Hertweck, F. Erdfelder, K.-A. Kreuzer, CD44 in hematological neoplasias, Ann Haematol. 90 (2011) 493-508.

[52] R. Stauder, M. Van Driel, C. Schwarzler, J. Thaler, H.M. Lokhorst, E.D. Kreuser, et al., Different CD44 splicing patterns define prognostic subgroups in multiple myeloma, Blood. 88 (1996) 3101-3108.

[53] S.K. Kumar, H. Uno, S.J. Jacobus, S.A. Van Wier, G.J. Ahmann, K.J. Henderson, et al., Impact of gene expression profiling-based risk stratification in patients with myeloma receiving initial therapy with lenalidomide and dexamethasone, Blood. 118 (2011) 4359-4362.

[54] M. Weinstock, Y. Aljawai, E.A. Morgan, J. Laubach, M. Gannon, A.M. Roccaro, et al., Incidence and clinical features of extramedullary multiple myeloma in patients who underwent stem cell transplantation, $\mathrm{Br} J$ Haematol. 169 (2015) 851-858.

[55] A.S. Azmi, B. Bao, F.H. Sarkar, Exosomes in cancer development, metastasis, and drug resistance: a comprehensive review, Cancer Metast Rev. 32 (2013) 623-642. 
739 [56] C. Kahlert, R. Kalluri, Exosomes in tumor microenvironment influence cancer progression and metastasis, J Mol Med. 91 (2013) 431-437. 
FIGURE CAPTIONS

742

743

744

745

746

747

748

749

750

751

752

753

754

755

756

757

758

759

760

761

762

763

764

765

766

767

768

769

770

771

772

773

774

775

776

777

778

779

780

781

782

783

784

785
Figure 1: $\quad \mathrm{MM}$ cell line EVs are enriched for $\mathrm{MHCl}$ and $\boldsymbol{\beta}_{2}-\mathrm{MG}$. A) Cryo-TEM images of vesicles derived from MM.1R and RPMI-8226 cells. B) Mean size distributions for vesicles derived from U266, MM.1S, MM.1R and RPMI-8226 cell lines by Nanosight analysis (bars indicate $95 \% \mathrm{Cl}$ ). C) 4way Venn diagram of vesicle protein identifications from MM cell lines. D) Expanded cluster map of extracellular vesicle and cellular lysate spectral counts for $\mathrm{MHCl}, \beta 2-\mathrm{MG}$ and GAPDH from MM cell lines E) Immunoblot of extracellular vesicle and cellular lysates for $\mathrm{MHCl}, \beta 2-\mathrm{MG}$ and GAPDH from $M M$ cell lines. Ponceau $S$ is provided for loading comparison. Collectively, these data show MM cell line derived vesicles are enriched in $\mathrm{MHCl}$ and $\beta_{2}-\mathrm{MG}$.

Figure 2: $\quad$ MM Patient EVs are enriched for $\mathrm{MHCl}$ and $\boldsymbol{\beta}_{2}$-MG. A) Mean serum vesicle size distributions from healthy donors $(n=3)$ and MM patients of different stages (MGUS $n=3$, Smoldering $n=8$, Active $n=3$, error bars indicate $95 \% \mathrm{Cl}$ ). B) Venn diagram of overlapping protein identifications from MM serum, MM bone marrow aspirates and healthy donor peripheral blood samples (whole blood \& leukopaks). C) Immunoblot for $\beta_{2}-M G$, $\mathrm{MHCl}$ and GAPDH from serum of representative random healthy donors (lanes 1-4) and MM patients (lanes 5-8). Ponceau S is provided for loading comparison. Collectively, these results illustrate an enrichment of $\mathrm{MHCl}$ and $\beta_{2}-\mathrm{MG}$ in the $\mathrm{MM}$ ex vivo EV samples when compared to healthy donor EVs.

Figure 3: Corticosteroid resistant cell lines harbor EVs with proteins associated drug resistance. A) A cluster map of 403 extracellular vesicle and cellular lysate protein spectral counts from the resistant (MM.1R) and parent (MM.1S) cell lines with at least one observation in each replicate of greater than five spectral counts. B) Expanded cluster maps of CD44, $\beta_{2^{-}}$ $M G$ and GAPDH from the MM.1R and MM.1S data. C) Immunoblot for CD44, $\beta_{2}-M G$, Lamin B1 and GAPDH from extracellular vesicle and cellular lysates. D) Flow cytometry for surface expression of CD44 from the MM cell lines. Collectively, these data show an enrichment of CD44 in the corticosteroid resistant cell line MM.1R EVs compared the parent MM.1S cell line.

Figure 4: Identification of the extracellular vesicles as the primary location of serum CD44 in MM patients. A) Immunoblot for CD44 from whole serum of healthy individuals and MM patients. B) Immunoblot for CD44 from whole serum (+) and vesicle depleted serum (-) from those samples labeled 1 \& 2 in Figure 4A. C) ELISA for CD44 from whole serum and vesicle depleted serum from those samples labeled $1 \& 2$ in Figure 4A. Collectively, these results show CD44 is primarily located in the serum vesicles of MM patients. 
Figure 5: Identification of serum CD44 as a predictor of overall survival in MM patients. A) ELISA for CD44 from 13 non-cancer donor age matched serum. B) ELISA for CD44 from MM patient serum in 233 newly diagnosed MM patients. C) Multivariable model for overall survival, HR: hazard ratio, 95\% Cl: 95\% confidence interval. D) Kaplan Meier survival curve for those patients with serum CD44 concentrations greater than 280 $\mathrm{ng} / \mathrm{mL}$. Collectively, these data show serum CD44 levels predict overall survival in multiple myeloma patients. 


\section{FIGURE 1}

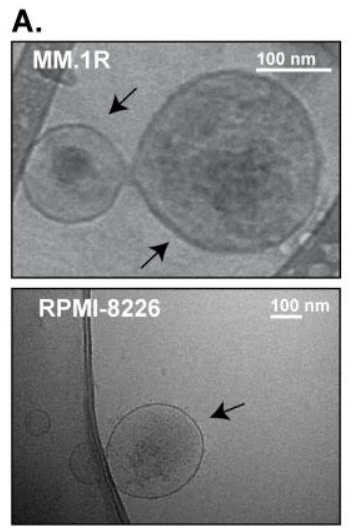

D.

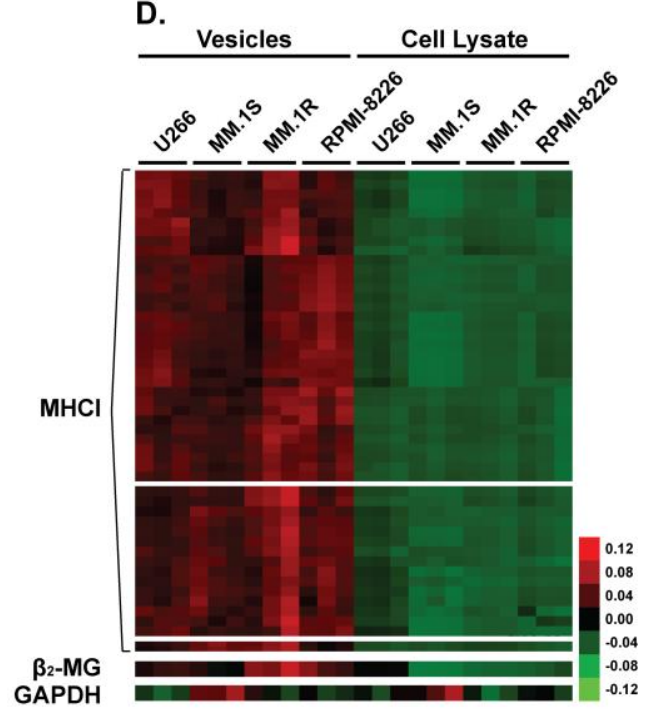

B.

Vesicle Size Distribution by Nanosight

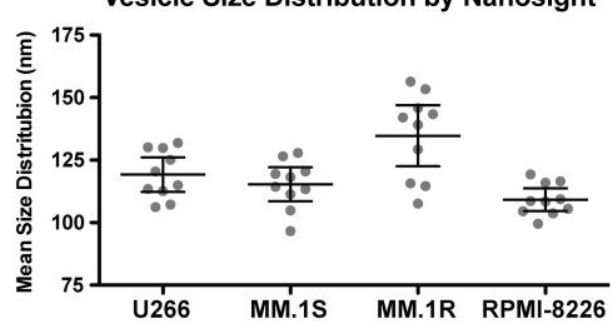

C.

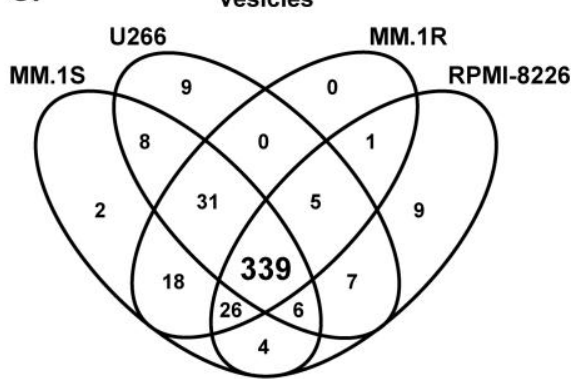

E.

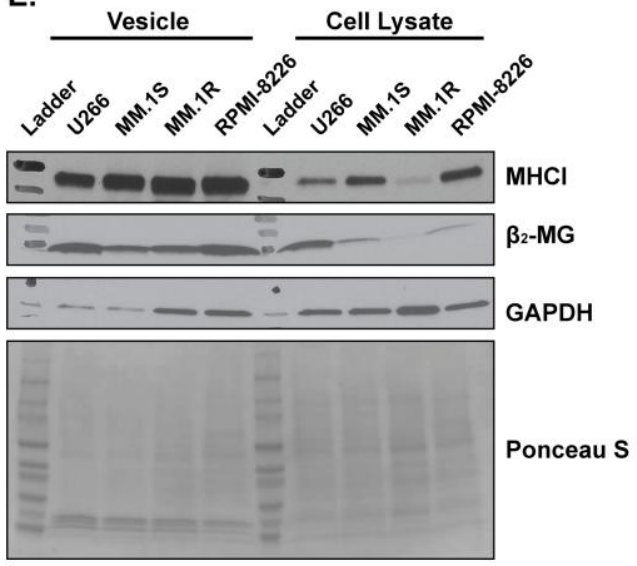



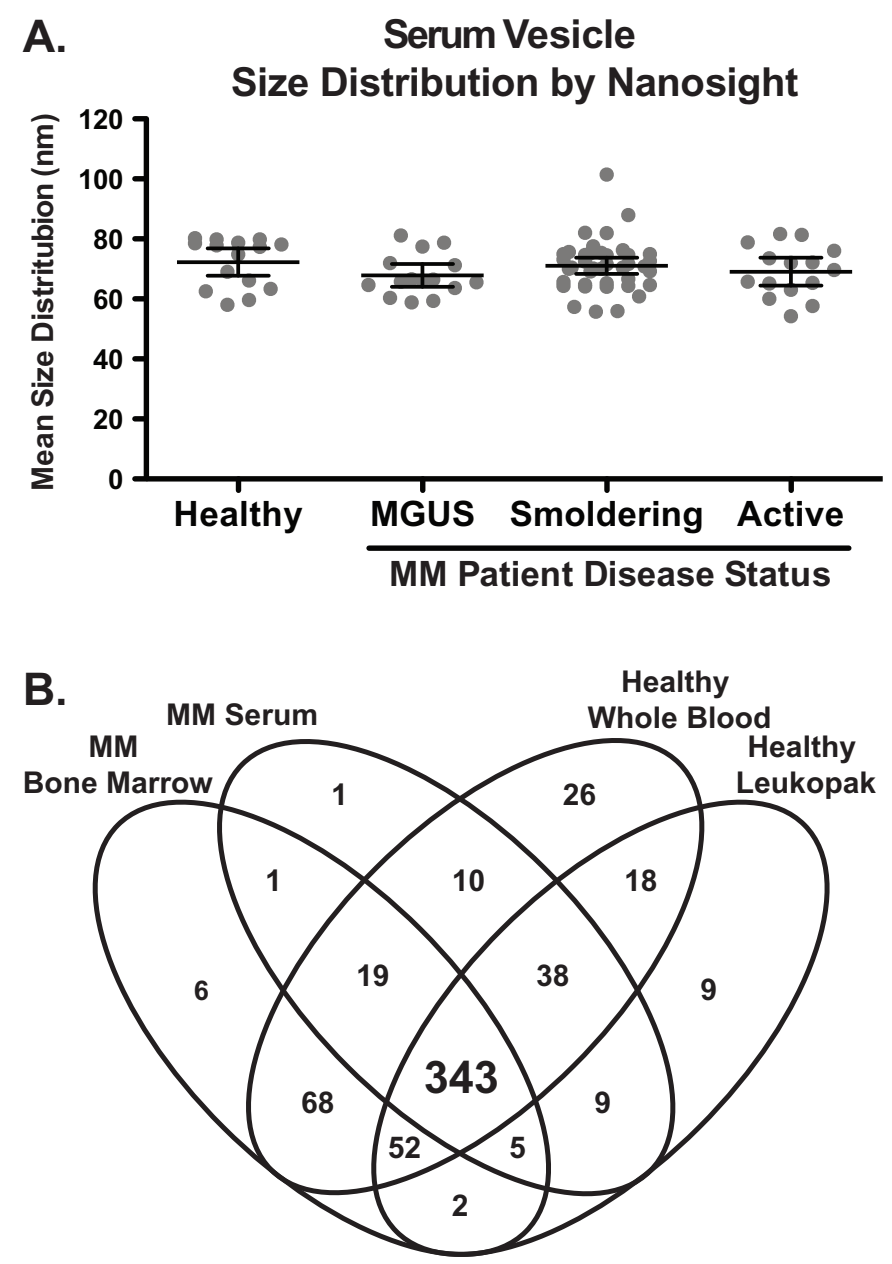

C. Serum Vesicles

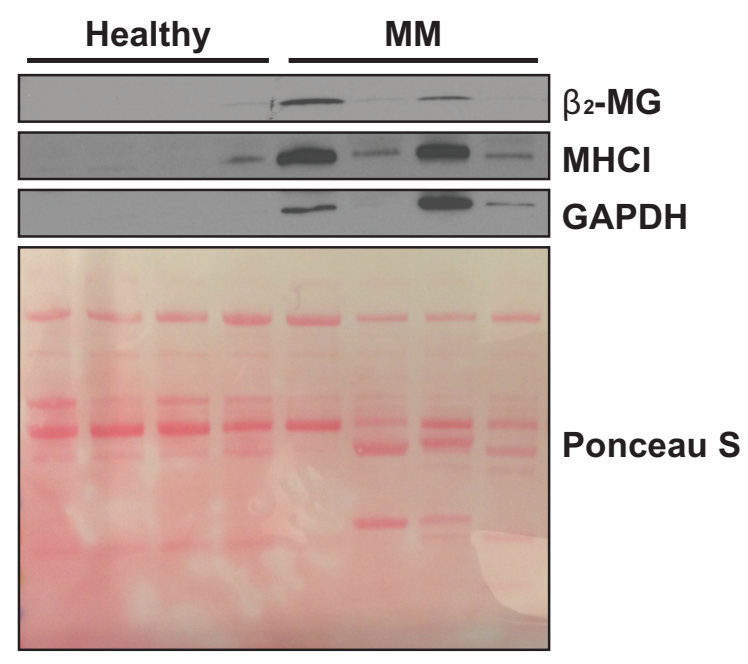


A.

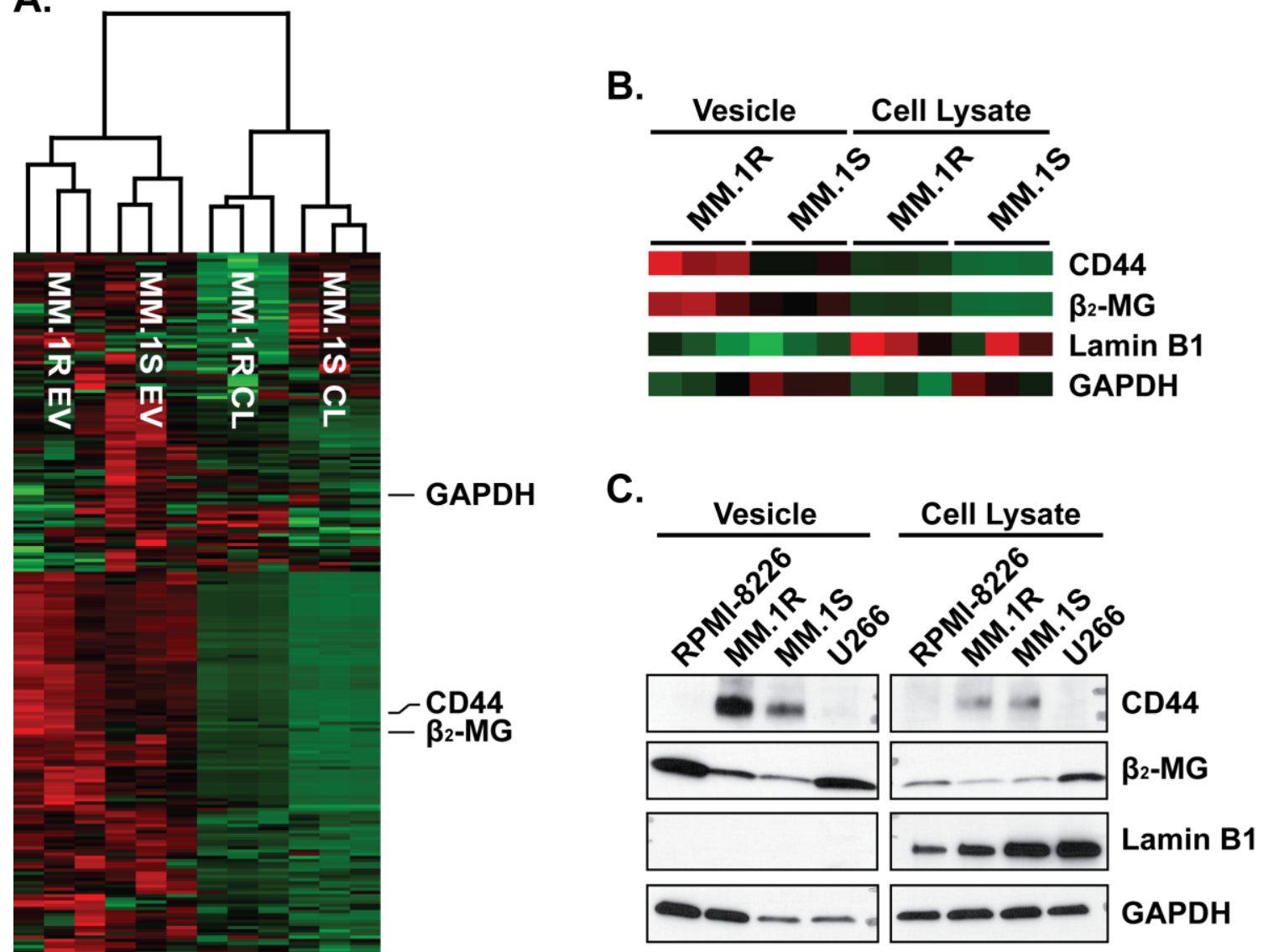

D.

- Lamin B1
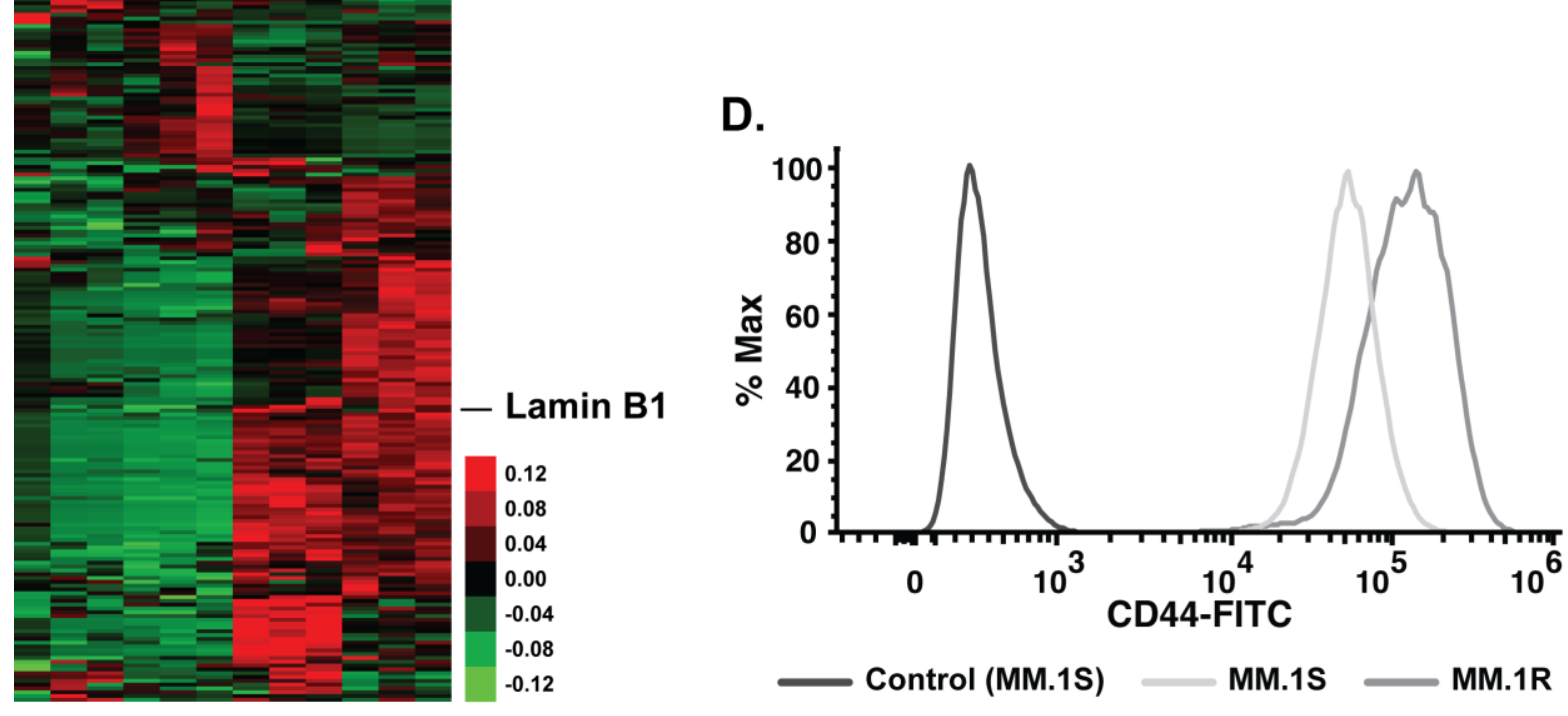


\section{FIGURE 4}

A.

Healthy Multiple Myeloma

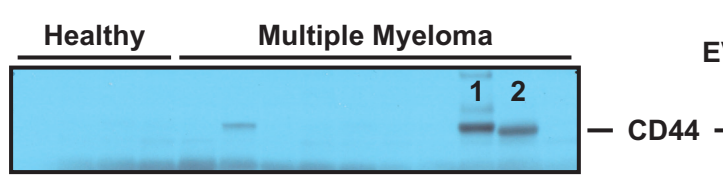

Whole Serum
B.

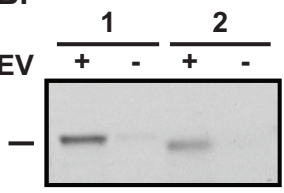

Whole Serum
C. $\overline{\overline{\mathrm{g}}} 180, \square \mathrm{EV}$

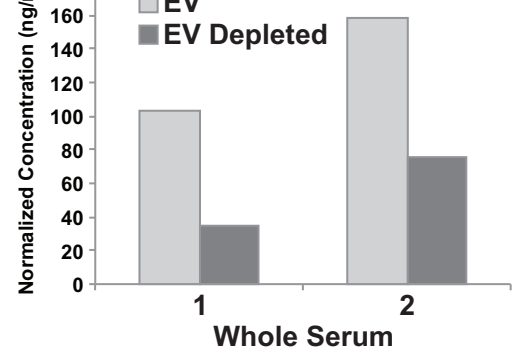

803 


\section{FIGURE 5}

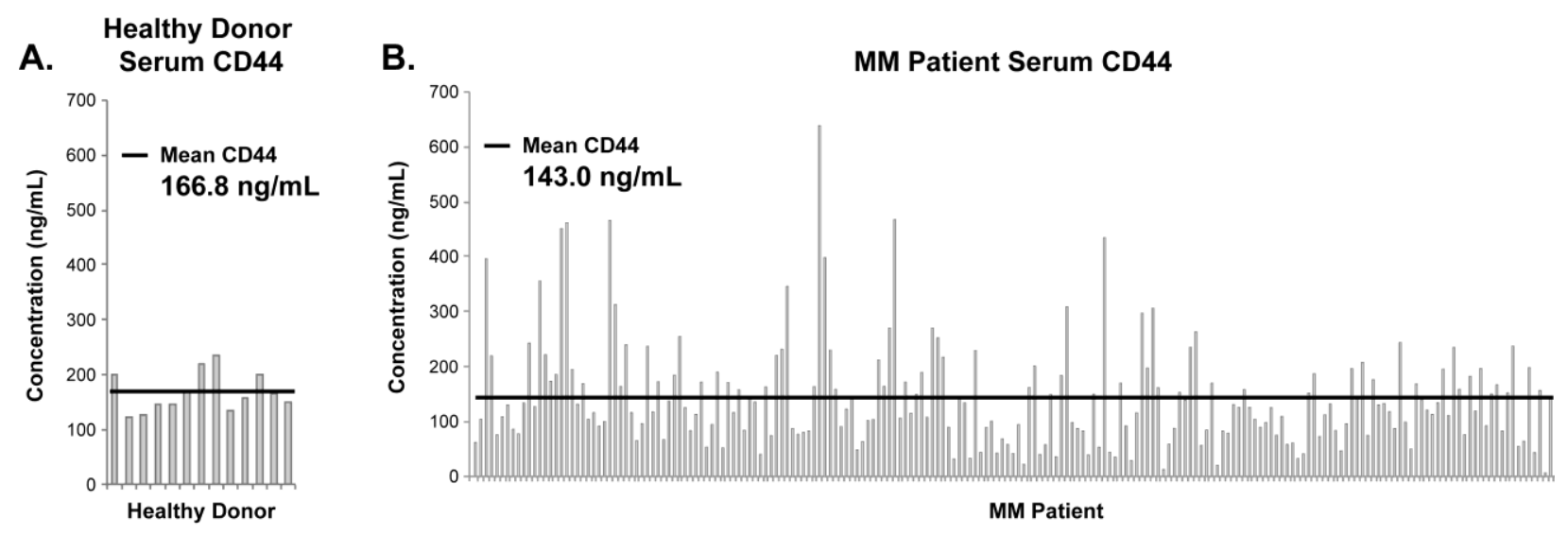

C. Multivariable Model for Overall Survival

Baseline

Characteristic

ISS Stage

II

sotype

BJ

$\lg A$

$\lg G$

FISH Risk

high

$\beta_{2}$-microglobulin

$<3.5$

3.55-5.5

$\geq 5.5$

CD44

$\leq 280 \mathrm{ng} / \mathrm{mL}$

$>280 \mathrm{ng} / \mathrm{mL}$

Creatinine

$<1.4$

$\geq 1.4$
HR $\quad 95 \% \mathrm{Cl}$ p-value

0.020

1.00

$2.16 \quad 0.92-5.09$

$3.38 \quad 1.42-8.04$

0.305

1.00

$1.73 \quad 0.72-4.15$

$1.16 \quad 0.51-2.63$

0.382

$0.77 \quad 0.44-1.38$

1.00

$1.51 \quad 0.74-3.11$

$2.33 \quad 1.19-4.58$

1.00
3.82

$3.82 \quad 1.85-7.91$

1.00

$1.41 \quad 0.78-2.55$
$<0.001$

0.252
0.048
D. Overall Survival

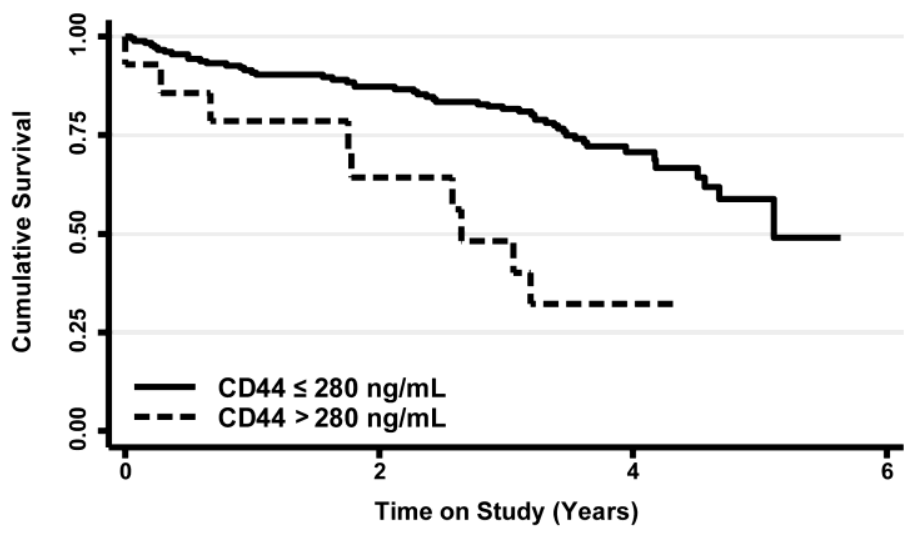

Time on Study (Years)
D. Overall Survival

$0.78-2.55$ 

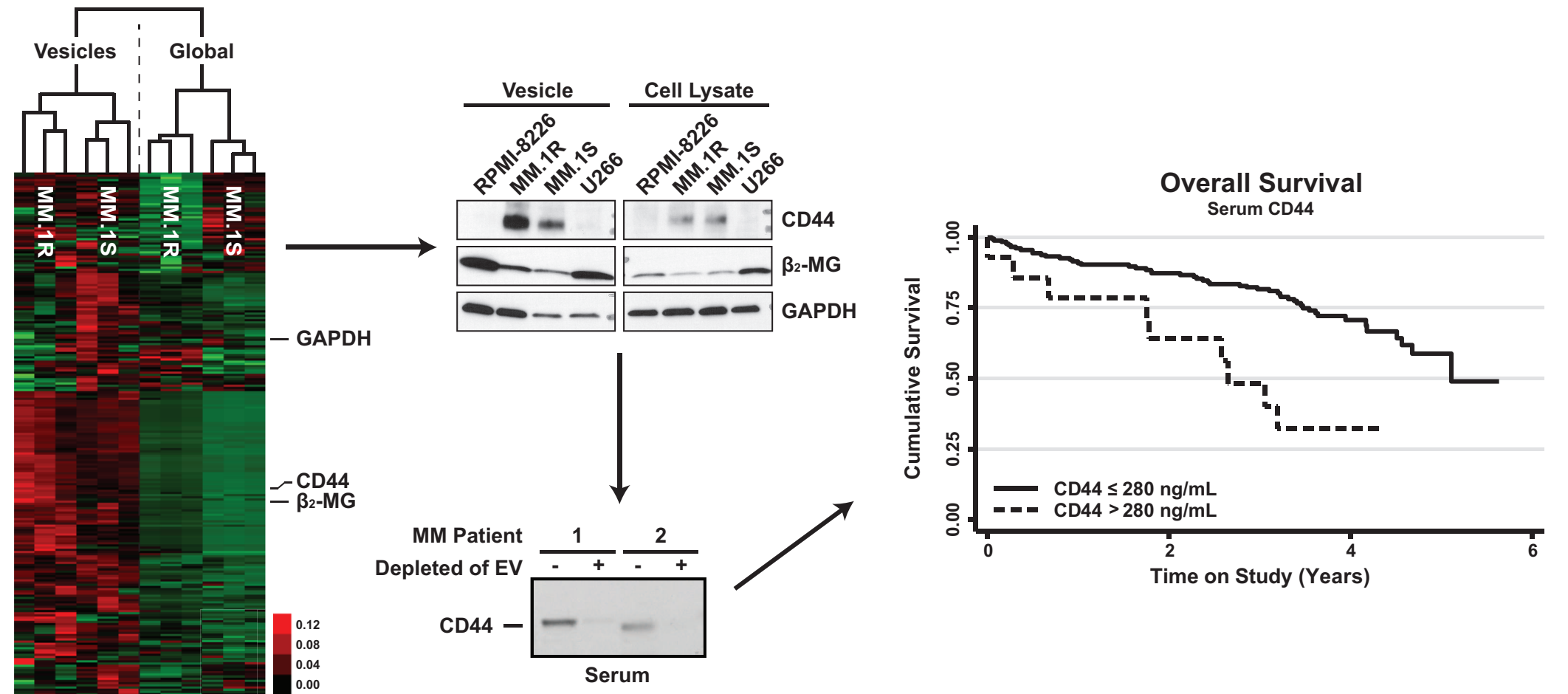